\title{
Comparison of surveillance-based metrics for the assessment and monitoring of disease detection: simulation study about type 2 diabetes
}

\author{
Ralph Brinks ${ }^{1,2^{*}}$, Annika Hoyer ${ }^{1}$, Deborah B. Rolka ${ }^{3}$, Oliver Kuss ${ }^{1,4}$ and Edward W. Gregg ${ }^{3}$
}

\begin{abstract}
Background: Screening and detection of cases are a common public health priority for treatable chronic conditions with long subclinical periods. However, the validity of commonly-used metrics from surveillance systems for rates of detection (or case-finding) have not been evaluated.

Methods: Using data from a Danish diabetes register and a recently developed illness-death model of chronic diseases with subclinical conditions, we simulate two scenarios of different performance of case-finding. We report different epidemiological indices to assess case-finding in both scenarios and compare the validity of the results.

Results: The commonly used ratio of detected cases over total cases may lead to misleading conclusions. Instead, the ratio of undetected cases over persons without a diagnosis is a more valid index to distinguish the quality of case-finding. However, incidence-based measures are preferable to prevalence based indicators.

Conclusion: Prevalence-based indices for assessing case-finding should be interpreted with caution. If possible, incidence-based indices should be preferred.
\end{abstract}

Keywords: Compartment model, Incidence, Prevalence, Diabetes, Chronic disease, Undiagnosed disease, Case-finding, Screening

\section{Background}

Chronic conditions like coronary heart disease, type 2 diabetes, hypertension, cancer, osteoporosis, and dementia frequently have long periods wherein the condition is undiagnosed. Although the specific policies related to active population screening are sometimes controversial, the prevalence of these undiagnosed conditions can be substantial and the period of undiagnosis is sometimes a missed opportunity to implement preventive care to reduce the risk of subsequent morbidity. Because of the lack of direct data on case-finding, population surveys with information on prevalence of diagnosed and

\footnotetext{
*Correspondence: Ralph.Brinks@ddz.uni-duesseldorf.de

${ }^{1}$ German Diabetes Center, Leibniz Institute for Diabetes Research at the

Heinrich-Heine-University Düsseldorf, Institute for Biometry and Epidemiology, Auf'm Hennekamp 65, 40225 Düsseldorf, Germany

${ }^{2}$ University Hospital at the Heinrich-Heine-University Düesseldorf, Hiller Research Unit for Rheumatology, Moorenstrasse 5, 40225 Düsseldorf, Germany

Full list of author information is available at the end of the article
}

undiagnosed cases are often incorporated into indirect indices to make inferences about levels of case-finding that are occurring. For example, national monitoring of public health efforts in the U.S. includes the tracking of proportion of cases of total diabetes [1], chronic kidney disease [2], and hypertension [3], who are aware of their condition or have been diagnosed. Similar metrics are used in diverse international settings to assess the degree of awareness, treatment and diagnosis of hypertension [4], hyperlipidemia [5], and diabetes [6]. Although these metrics are intended to assess the degree of case detection that occurs in clinical and public health settings, the validity of these surrogates of the rate of case-finding has not been evaluated. In this analysis we examine common approaches to using cross-sectional data and find that the choice of metric can yield vastly different conclusions about trends in detection, with some yielding misleading conclusions. 
In this article, we use a recently developed multi-state model and simulate two different scenarios about diabetes case-finding. Then, we apply and compare different metrics to assess the case-finding in the two scenarios.

\section{Methods}

Based on data from the national Danish diabetes register on diagnosed diabetes, we simulate two different scenarios about case-finding, in one scenario the prevalence of undetected disease increases over time, and in another scenario the prevalence of undetected disease decreases over time. For brevity, we will denote the two scenarios as $\mathrm{PU}^{+}$and $\mathrm{PU}^{-}$. Here, $\mathrm{PU}$ means prevalence of undiagnosed disease, and the + and - signs indicate the upward and downward trend in time, respectively.

For the simulation we use a previously published multistate model. We then apply different surveillance-based indices to the $\mathrm{PU}^{-}$and $\mathrm{PU}^{+}$scenarios and compare the results of different indices.

After a brief description of the multi-state model, we describe the different indices for assessing case-finding. Then, details of the simulation are presented.

\section{Multi-state model}

Recently, we described an illness-death model with a state of being Undiagnosed preceding the Diagnosed state (Fig. 1) [7]. At birth, each person is in the Normal state (here we just consider diseases acquired after birth). During the life course, the person may contract the disease and enter the Undiagnosed state. In a screening program, or as the disease becomes symptomatic, or during some routine medical examinations, the disease may be diagnosed and the person transitions to the Diagnosed state. Persons may die during any of these three states. Since we are considering chronic conditions only, we assume that backward steps from the Undiagnosed state to the Normal

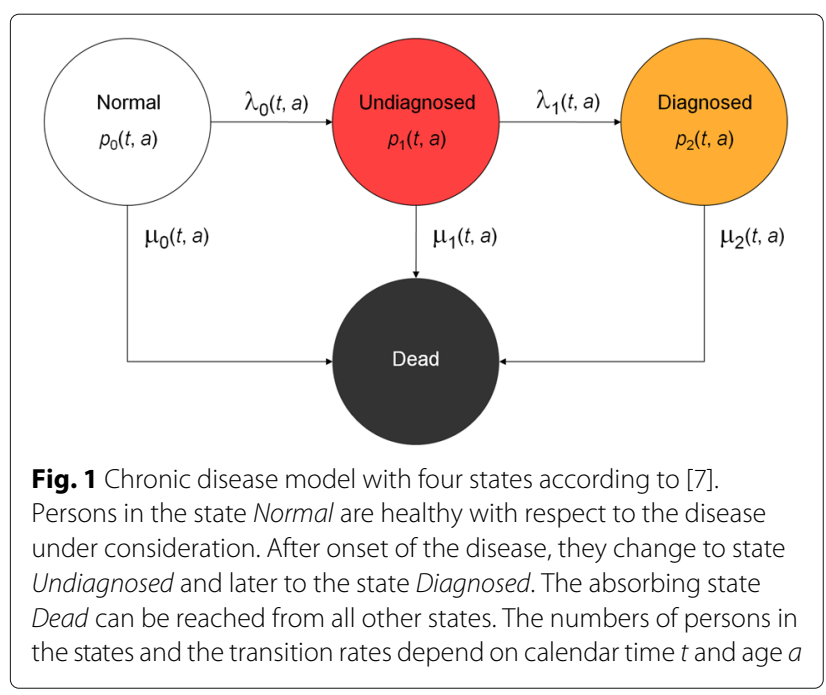

state are not possible. Similarly, there are no backward steps from the Diagnosed to the Undiagnosed state.

The transition rates $\lambda_{\ell}(t, a), \ell=0,1$, and $\mu_{k}(t, a), k=$ $0,1,2$, in the model and the percentages of persons in the states, the prevalences, are related by a two-dimensional system of partial differential equations [7]. As in [7] let $p_{k}(t, a)$ denote the fraction of persons aged $a$ at time $t$ in state $k$. In epidemiological settings time $t$ is also called period. For example, $p_{2}(t, a)$ is the fraction of persons in the population who are aged $a$ at time $t$ and are in the Diagnosed state $(k=2)$. It can be calculated as $p_{2}(t, a)=\frac{N_{2}(t, a)}{N(t, a)}$ where $N_{k}(t, a)$ denotes the number of people in state $k, k=0,1,2$, aged $a$ at time $t$ and $N(t, a)=$ $N_{0}(t, a)+N_{1}(t, a)+N_{2}(t, a)$. Similary, the prevalence of the undiagnosed disease $\left(p_{1}\right)$ is defined as $p_{1}(t, a)=\frac{N_{1}(t, a)}{N(t, a)}$.

The system of partial differential equations governing the compartment model shown in Fig. 1 is:

$$
\left(\frac{\partial}{\partial t}+\frac{\partial}{\partial a}\right) p_{1}=\lambda_{0}\left(1-p_{2}\right)-\left(\lambda_{0}+\lambda_{1}+\mu_{1}-\mu_{0}\right) p_{1}+p_{1} z
$$

$$
\left(\frac{\partial}{\partial t}+\frac{\partial}{\partial a}\right) p_{2}=-\left(\mu_{2}-\mu_{0}\right) p_{2}+\lambda_{1} p_{1}+p_{2} z
$$

where $z=p_{1}\left(\mu_{1}-\mu_{0}\right)+p_{2}\left(\mu_{2}-\mu_{0}\right)$.

The rate $\lambda_{0}$ can be interpreted as the true incidence or total incidence of type 2 diabetes. It describes how many persons develop diabetes in the considered population irrespective of whether diabetes is diagnosed or not. The total incidence $\lambda_{0}$ is affected by the aetiologic risk profile of the population. If risk factors become more prevalent in the considered population over time, the rate $\lambda_{0}$ will increase accordingly. Vice versa, if risk factors become less frequent, $\lambda_{0}$ will go down.

The rate $\lambda_{1}$ describes the transition from the undiagnosed state to the diagnosed state. While $\lambda_{0}$ is affected by aetiological factors, $\lambda_{1}$ mainly depends on societal factors, e.g., medical progress in detecting the disease, awareness of patients and physicians, reimbursement of diagnostic testing etc. Epidemiologists are interested in both rates, $\lambda_{0}$ as it reflects the risk profile of a population and $\lambda_{1}$ as a societal construct. However, if an epidemiologist refers to "the incidence" of a chronic disease and the undiagnosed state is ignored, this "incidence" essentially refers to $\lambda_{1}$. Hence, it allows little inference about the changes of risk profiles in the population.

\section{Indices for assessing case-finding}

For better clarity, we categorize the indices on whether they are based on prevalence or transition rates in the multi-state model. The last category refers to an index, which is combined from prevalence and transition rates. 


\section{Indices based on the prevalence}

The simplest index is the prevalence of the undiagnosed disease $\left(p_{1}\right)$. A high or an increasing value of $p_{1}$ is intuitively considered to be unfavourable. For example, if $p_{1}$ is $5 \%$ in a specific age group at a specific point in time, and is $10 \%$ two years later, then there is an (unfavourable) accumulation of undetected cases during these two years.

The second index to approach case-finding, is the proportion of detected cases from the total cases [1], i.e.,

$$
\omega_{1}=\frac{p_{2}}{p_{1}+p_{2}} .
$$

The reciprocal of $\omega_{1}$ describes the factor the diagnosed cases have to be multiplied with to obtain the number of all cases of the chronic disease. If $\omega_{1}$ equals 0.5 , for instance, this means that for each detected case there is one undetected case. Obviously, it holds $0 \leq \omega_{1} \leq 1$. A high value in $\omega_{1}$ is usually interpreted as advantageous with respect to case-finding [1].

Similarly, it may be useful to consider the proportion

$$
\omega_{2}=\frac{p_{1}}{p_{0}+p_{1}}
$$

This proportion relates the number of persons in the undiagnosed state to all persons who do not have a diagnosis, i.e., the healthy and the undiagnosed. The idea behind the measure $\omega_{2}$ is that case-finding can be thought of the task of distinguishing persons from a pool consisting of healthy and undiagnosed persons. This pool of healthy and undiagnosed persons may be seen as the search space. The search space is subject to the activities of case-finding. Once an undiagnosed person is identified as a case, this person gets a diagnosis and is removed from the search space henceforth. As the disease under consideration is chronic, there is no way back into the search space, i.e., no remission can occur. In contrast to $\omega_{1}$, the figure $\omega_{2}$ just refers to the persons who are at risk for a possible diagnosis. Thus, the fraction of persons with a diagnosis does not play a role for $\omega_{2}$. The reciprocal of $\omega_{2}$ is the average number of persons without a diagnosis a physician must see to meet one undiagnosed case.

Again, it holds $0 \leq \omega_{2} \leq 1$. Ideally, $\omega_{2}$ is 0 , i.e., all undetected cases become diagnosed and are removed from the search space. The closer $\omega_{2}$ approaches 1 , the more the search space is dominated by the undiagnosed persons. Thus, a lower value of $\omega_{2}$ is advantageous in assessing case-finding. This is consistent with the interpretation of $\omega_{2}$ being the reciprocal of the average number of persons without diagnosis a physician must meet to have one undiagnosed case. The lower $\omega_{2}$ is, the greater the reciprocal is. Hence, a lower $\omega_{2}$ implies a higher average number a physician must meet to find an undiagnosed case. This means, case-finding worked well in the time before the physician met these persons.

\section{Indices based on the transition rates}

Apart from the indices based on the prevalences, we may consider figures based on the transitions in the model. In [7] we used the detection rate ratio $D R=\frac{\lambda_{1}}{\lambda_{0}}$, which relates the instantaneous risk (hazard) of transitioning to the diagnosed state to the risk of becoming an undetected case. As it is unlikely to be diagnosed immediately after entering the undiagnosed state, we introduce a delay parameter $\gamma, \gamma \geq 0$, and define

$$
\operatorname{DR}_{\gamma}(t, a)=\frac{\lambda_{1}(t+\gamma, a+\gamma)}{\lambda_{0}(t, a)} .
$$

It holds $\mathrm{DR}=\mathrm{DR}_{0}$, and in [7] we have shown that $\mathrm{DR}$ determines if the prevalence of the undiagnosed disease is lowering or rising. The condition

$$
\mathrm{DR}(t, a)<\frac{p_{0}(t, a)}{p_{1}(t, a)}+\frac{\mu(t, a)-\mu_{1}(t, a)}{\lambda_{0}(t, a)}
$$

is equivalent with increasing $p_{1}$ in $(t, a)$, i.e., $p_{1}(t, a)<$ $p_{1}(t+\delta, a+\delta)$ for small $\delta>0$.

Another important index is the fraction of healthy persons aged $a$ at time $t$ who become incident undiagnosed cases at time $t$ and die within $\gamma>0$ time units without ever obtaining a diagnosis. As these persons do not have a diagnosis, they never were treated. To develop this figure, we first calculate the probability of dying $P_{\gamma}^{(\text {dead })}$ during the first $\gamma>0$ time units in the undiagnosed state:

$$
\begin{aligned}
P_{\gamma}^{(\text {dead })}(t, a) & =\int_{0}^{\gamma} \mu_{1}(t+s, a+s) \\
& \exp \left(-\int_{0}^{s}\left(\lambda_{1}+\mu_{1}\right)(t+\tau, a+\tau) \mathrm{d} \tau\right) \mathrm{d} s .
\end{aligned}
$$

The probability $P_{\gamma}^{(\mathrm{dead})}$ is combined with the incidence rate $\lambda_{0}$ :

$$
\Phi_{\gamma}(t, a)=\lambda_{0}(t, a) P_{\gamma}^{(\mathrm{dead})}(t, a) .
$$

Then, $\Phi_{\gamma}(t, a)$ is the number of persons aged $a$ at time $t$, who become incident undiagnosed cases at $t$ and die within $\gamma$ time units without diagnosis. These originally healthy persons never had the chance of obtaining a treatment.

\section{Composite indices}

In the field of infectious disease epidemiology, the case detection rate (CDR) is defined as the notification rate of incident cases over the total incidence rate. Roughly speaking, it is the proportion of detected incident cases from the total incident cases [8]. In our multi-state model, the total incidence is $\lambda_{0}$ and the CDR can be calculated as:

$$
\mathrm{CDR}=\mathrm{DR}_{0} \times \omega_{2}
$$


A proof for this relation can be found in the Appendix. As the CDR includes the transition rate ratio $\mathrm{DR}_{0}$ and the prevalence-based index $\omega_{2}$, we call CDR a composite index for assessing case-finding.

Table 1 sums up the different indices for assessing casefinding.

\section{Simulation}

Carstensen and co-workers have described an increase of the incidence of diagnosed diabetes in Denmark during the second half of the 1990ies [9]. The data this estimate was based upon stem from a nationwide diabetes register with a catchment population of more than 5 million people. The data about diagnosed diabetes from Denmark are very comprehensive and very detailed. Unfortunately, there are no data of similar high quality and details about undiagnosed diabetes for Denmark during that time. So, we use the rates reported in [9] and additional assumptions to include the state of undiagnosed diabetes in our simulation. The scenarios are hypothetical, because the Danish register does not report data about undiagnosed diabetes. The aim of this work is not to state that the situation of undiagnosed diabetes in Denmark has been as we describe, we are only interested in simulating somewhat realistic situations that could have been. Our aim is the exploration and comparison of how the indices of case-finding perform in these scenarios. We confine ourselves to the data from the male Danish population.

Together with an initial condition, the simulation uses the rates $\lambda_{\ell}, \ell=0,1$, and $\mu_{k}, k=0,1,2$ as described below and integrates Eqs. (1) and (2) to obtain the functions $p_{k}, k=1,2$. We will numerically integrate the system (1)-(2) using the Method of Characteristics [10] and Runge-Kutta numerical integration [11]. After numerically integrating system (1)-(2),

Table 1 Summary of all indices for assessing case-finding

\begin{tabular}{|c|c|c|}
\hline Index & Formula & Remark \\
\hline$\omega_{1}$ & $\frac{p_{2}}{p_{1}+p_{2}}$ & $\begin{array}{l}\text { Percentage of diagnosed over } \\
\text { total cases }\end{array}$ \\
\hline$\omega_{2}$ & $\frac{p_{1}}{p_{0}+p_{1}}$ & $\begin{array}{l}\text { Inverse of the average number } \\
\text { of persons without a diagnosis } \\
\text { whom a physician must see in } \\
\text { order to meet one undiagnosed } \\
\text { case }\end{array}$ \\
\hline $\mathrm{DR}_{\gamma}(t, a)$ & $\frac{\lambda_{1}(t+\gamma, a+\gamma)}{\lambda_{0}(t, a)}$ & $\begin{array}{l}\text { Rate ratio of diagnosing a } \\
\text { person exactly } \gamma \text { years after } \\
\text { contracting the disease }\end{array}$ \\
\hline CDR & $\mathrm{DR}_{0} \omega_{2}$ & Case detection ratio \\
\hline$\Phi_{\gamma}(t, a)$ & $\lambda_{0}(t, a) P_{\gamma}^{(\mathrm{dead})}(t, a)$ & $\begin{array}{l}\text { Number of healthy persons } \\
\text { aged } a \text { at } t \text { who die with at most } \\
\gamma \text { years of undiagnosed disease }\end{array}$ \\
\hline
\end{tabular}

we apply the epidemiological indices that have been described above.

\section{Initial condition}

The age-specific prevalence of diagnosed diabetes in the male Danish population in the year 1995 serves as the initial condition for $p_{2}$. Additionally, we assume that the prevalence of undiagnosed diabetes $\left(p_{1}\right)$ is half of $p_{2}$.

\section{Mortality}

The mortality rate $\mu_{0}$ in our simulation is chosen to be the mortality of the non-diabetic population in Denmark. Following [9], a yearly decrease of $2.5 \%$ for all ages is assumed. Because there is evidence that the all-cause mortality of men with undiagnosed diabetes is elevated by $25 \%$ as compared to normoglycemic men [12], we assume $\mu_{1}=$ $1.25 \mu_{0}$. The mortality of men with diagnosed diabetes $\left(\mu_{2}\right)$ is taken from the Danish register.

\section{Transition rate $\lambda_{0}$}

Detailed information about the incidence of diagnosed diabetes is reported by Carstensen et al. To include a state of undiagnosed diabetes, the transition rate $\lambda_{0}$ from the Normal to the Undiagnosed state is assumed to be twice as high as the incidence of diagnosed diabetes in [9] (left part of Fig. 2). Although the exact ratio between the incidence of undiagnosed and diagnosed diabetes has never been surveyed, the last assumption is based on the observation that in Denmark there is a considerable proportion of undiagnosed diabetes [13, 14]. Thus, the amount of overall diabetes is higher than diagnosed diabetes alone. The increase of $5.3 \%$ per year has again been taken from the Danish register data.

\section{Transition rate $\lambda_{1}$}

The rate $\lambda_{1}$ is chosen as a sigmoid function (right part of Fig. 2). It mimics an awareness for diabetes, which increases from age 15 until some saturation level at about 40 years of age. After an age of 40 years, the level remains constant. From year 1995 to year 2000, we assume that there is no annual trend in $\lambda_{1}$. During 2000-2005, for $\lambda_{1}$ we assume an annual change of $-5 \%$ and $+15 \%$ in the $\mathrm{PU}^{+}$ and the $\mathrm{PU}^{-}$scenarios, respectively. These choices are merely assumptions because we do not have any empirical data about $\lambda_{1}$.

Based on the compartment model shown in Fig. 1 and the data from the Danish register, the indices summarized in Table 1 are applied to the two scenarios $\mathrm{PU}^{+}$and $\mathrm{PU}^{-}$. Then, the results are compared. All calculations for this work have been performed with the statistical software R (The R Foundation for Statistical Computing). The R source files for running the simulation and applying the indices for assessing the case-finding are provided as an Additional file 1 to this manuscript. 

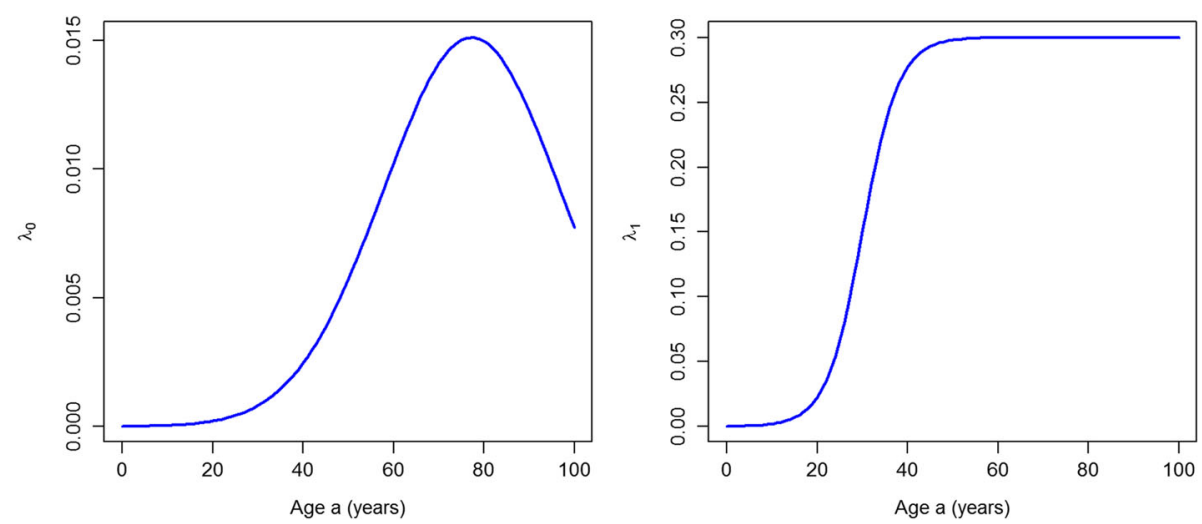

Fig. 2 Incidence rates. Age-specific transition rates $\lambda_{0}$ (left) and $\lambda_{1}$ (right) in year $t=1995$

\section{Results}

\section{Scenario of increasing prevalence of undiagnosed disease}

We will start with the $\mathrm{PU}^{+}$scenario. Figure 3 shows the age-specific prevalence of the undiagnosed $\left(p_{1}\right.$, left part of Fig. 3 ) and the diagnosed disease ( $p_{2}$, right part) in the years $t_{1}=2000$ (solid lines) and $t_{2}=2005$ (dashed lines). For all ages, there is an increase of the prevalence of undiagnosed diabetes from 2000 to 2005 . Thus, in the $\mathrm{PU}^{+}$ scenario there is an accumulation of undetected cases in the undiagnosed state. Similarly, for virtually all ages there is an increase of the diagnosed prevalence during the period 2000-2005. If we compare the age-specific prevalences for 2000 and 2005 in the right part of Fig. 3 with the corresponding prevalence data published in [9], we see a good agreement. Hence, the rates chosen for the $\mathrm{PU}^{+}$ scenario are consistent with the observed register data from Denmark.

If we calculate the prevalence-based indices of casefinding $\omega_{1}$ and $\omega_{2}$, we obtain the graphs shown in Fig. 4. The left part of Fig. 4 shows that $\omega_{1}$ indicates an improvement of case-finding between the years 2000 (solid line) and 2005 (dashed line) for the age range 30 to about 60 . For higher ages the two lines converge, which indicates no difference in the performance of case-finding.

The index $\omega_{2}$ (right part of Fig. 4) finds that case-finding worsens during the five years from 2000 to 2005 for all ages older than 40 years. For lower ages, the index does not show a difference between the years 2000 and 2005.

Figure 5 shows the indices based on the incidence rates. For the whole age range, both indices display a decreasing performance of case-finding during the period 2000 and 2005.

Similarly, the indices $\Phi_{5}$ and CDR (Fig. 6) show a decreasing performance of case-finding for all ages during 2000-2005. Note the large increase in the numbers of persons dying without ever obtaining a treatment. For example, consider 100,000 healthy persons aged 90 in year 2000. Of these, 1587 develop diabetes during that year. During the next five years, 795 (50\%) of these 1587 die without ever obtaining a diagnosis. In 2005, 2055 persons develop diabetes and 1064 (52\%) of these die without a diagnosis during the next five years.
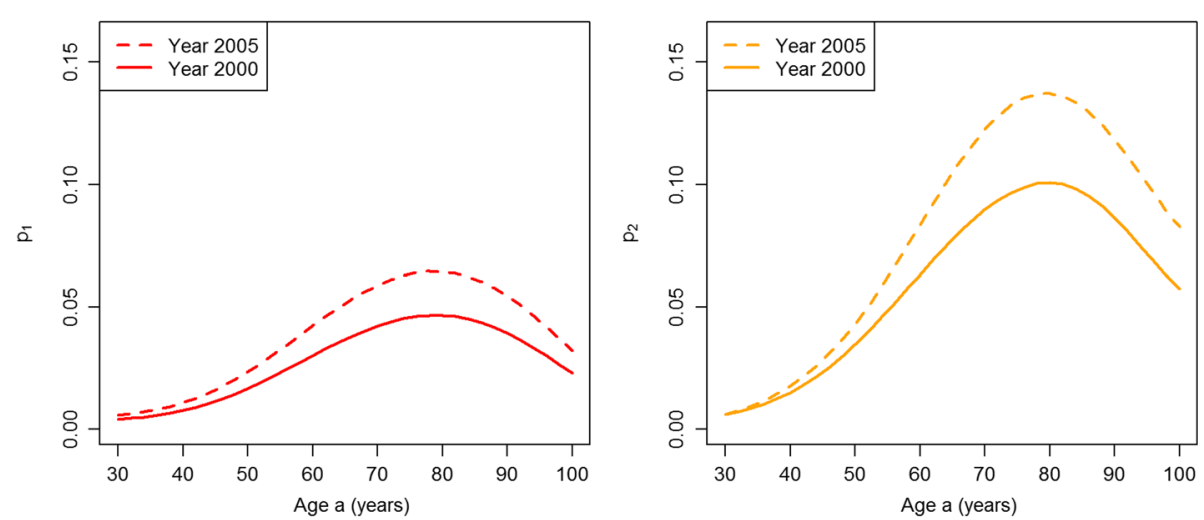

Fig. 3 Prevalences in the worsened case-finding scenario. Age-specific prevalences $p_{1}$ (left) and $p_{2}$ (right) in the years $t=2000$ (solid lines) and $t=2005$ (dashed lines) in the scenario $\mathrm{PU}^{+}$ 

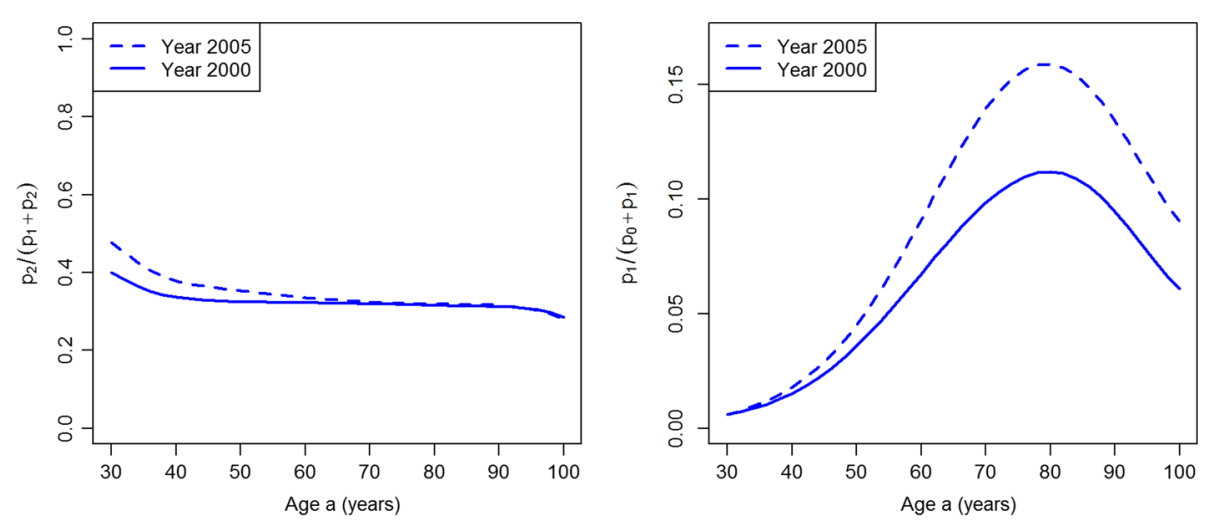

Fig. 4 Prevalence-based indices in the worsened case-finding scenario. Age-specific indices of case-finding $\omega_{1}$ (left) and $\omega_{2}$ (right) in the years $t=2000$ (solid lines) and $t=2005$ (dashed lines) in the scenario PU ${ }^{+}$

Scenario of decreasing prevalence of undiagnosed disease In the $\mathrm{PU}^{-}$scenario, we obtain the age-specific prevalences $p_{1}$ and $p_{2}$ in the years $t_{1}=2000$ (solid lines) and $t_{2}=2005$ (dashed lines) as shown in Fig. 7 .

For all ages above about 45 , there is a decrease of the prevalence of undiagnosed diabetes during the period from 2000 to 2005. The age-specific prevalences of undiagnosed and diagnosed diabetes in year 2000 agree with the corresponding curves in the $\mathrm{PU}^{+}$scenario (see Fig. 3). For virtually all ages, there is a decrease of the prevalence of undiagnosed diabetes during the period 2000-05. During the same period the prevalence of the diagnosed disease increases for all ages.

The prevalence-based indices $\omega_{1}$ and $\omega_{2}$ are shown in Fig. 8. Both indices $\omega_{1}$ and $\omega_{2}$ indicate an improvement of the quality case-finding.

The ratios $\mathrm{DR}_{0}$ and $\mathrm{DR}_{5}$ are presented in Fig. 9. For the whole age range, both indices $\mathrm{DR}_{0}$ and $\mathrm{DR}_{5}$ consistently display an increasing performance of case-finding during the considered period. The same holds true for the indices CDR and $\Phi_{5}$ (Fig. 10)
For comparison with the $\mathrm{PU}^{+}$scenario, consider 100,000 healthy persons aged 90 in year 2000. Again, 1587 develop diabetes during that year. During the next five years, $713(45 \%)$ of these 1587 die without obtaining a diagnosis. In 2005, from those 2055 persons who become diabetic in that year $566(28 \%)$ die without a diagnosis during the next five years. This is a substantial reduction, which indicates an improvement in case-finding.

Table 2 sums up the findings of assessing the performance of case-finding in the different simulations scenarios. The index $\omega_{1}$ at least partly contradicts the findings of the other indices in scenario $\mathrm{PU}^{+}$, whereas the figures $p_{1}, \omega_{2}, \mathrm{DR}_{0}, \mathrm{DR}_{5}$ and $\Phi_{5}$ assess both scenarios consistently. For some ages, the indices $p_{1}$ and $\Phi_{5}$ are indecisive.

\section{Discussion}

Based on a recently developed multi-state model, we simulated two hypothetical scenarios about trends in undiagnosed type 2 diabetes. In one scenario the incidence rate of diagnoses decreased over time reflecting a worsened
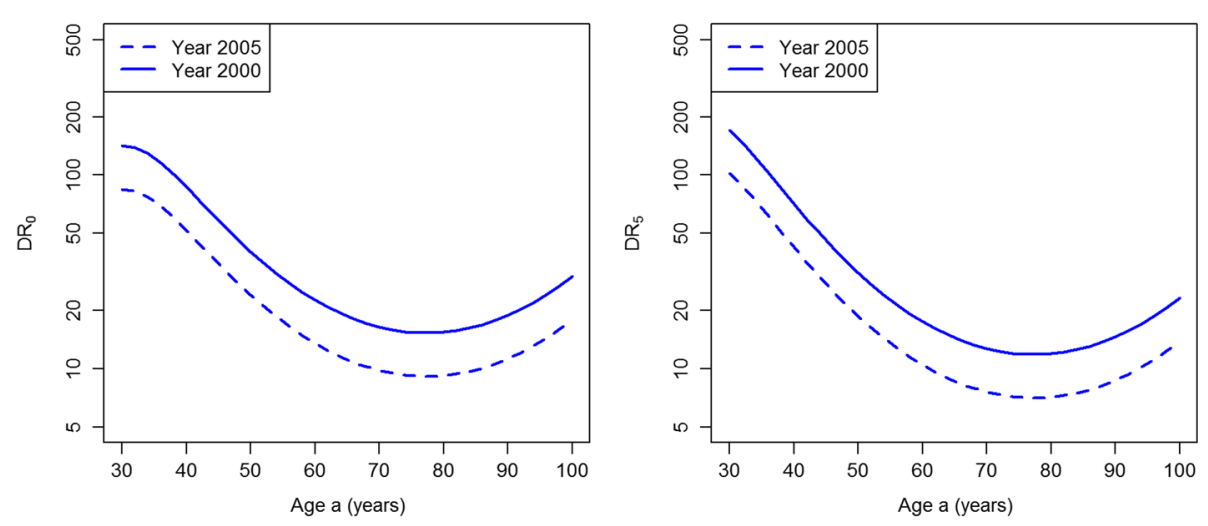

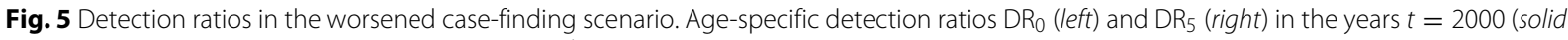
lines) and $t=2005$ (dashed lines) in the scenario $\mathrm{PU}^{+}$ 

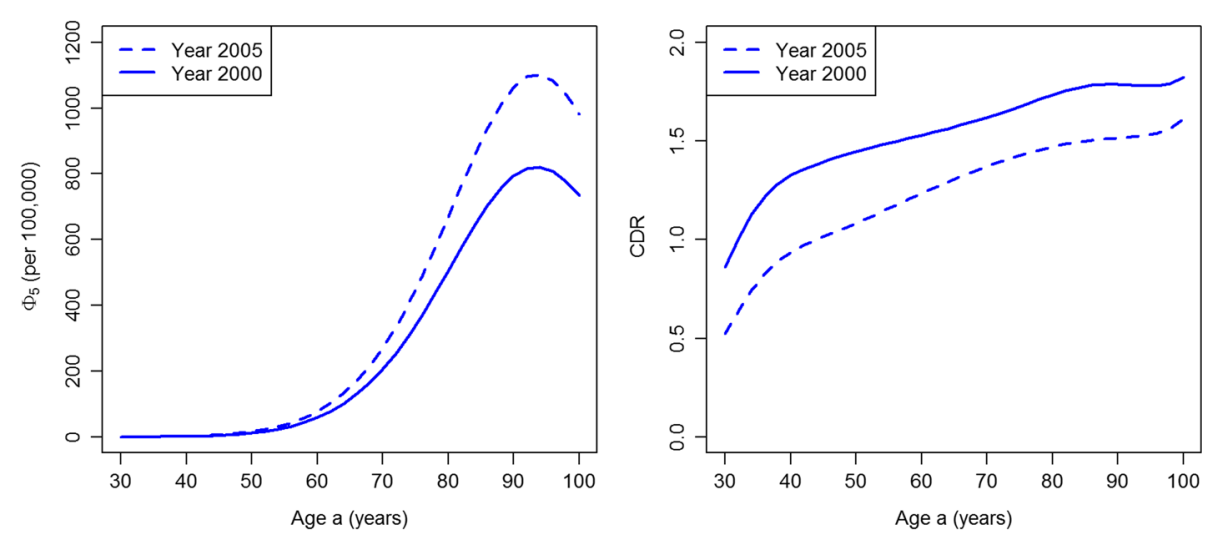

Fig. 6 Number of deaths from the undiagnosed state and case detection rate in the worsened case-finding scenario. Age-specific number $\Phi_{5}$ (per 100,000 healthy persons, left) and the case detection rate CDR (right) in the years $t=2000$ (solid lines) and $t=2005$ (dashed lines) in the scenario PU ${ }^{+}$

case-finding. In the other scenario, the performance of case-finding improved. Several indices for assessing casefinding have been applied to the two scenarios.

We found that the measure $\omega_{1}$ leads to an inconsistent assessment of the case-finding performance in our scenarios. In the scenario of increasing prevalence of undiagnosed disease $\left(\mathrm{PU}^{+}\right), \omega_{1}$ indicates an improvement whereas all other indices indicate a worsening of the quality of case-finding.

The index $\omega_{2}$ assesses both scenarios according to the incidence-based and composite indices. Thus, $\omega_{2}$ is a sensitive measure for the improvement and degradation of case-finding in our simulation. There is a theoretical reason for the appropriateness of $\omega_{2}$. As we have seen in Eq. (5), the expression $\frac{p_{0}}{p_{1}}+\frac{\mu-\mu_{1}}{\lambda_{0}}$ determines if $p_{1}$ is increasing or decreasing. This expression can be reformulated in terms of $\omega_{2}$ :

$$
\frac{p_{0}}{p_{1}}+\frac{\mu-\mu_{1}}{\lambda_{0}}=\frac{1}{\omega_{2}}-1+\frac{\mu-\mu_{1}}{\lambda_{0}}
$$

Thus, we can see that $\omega_{2}$ plays a direct role in determining if the prevalence of the undiagnosed disease is increasing or decreasing.

Similarly, the detection ratios $\mathrm{DR}_{\gamma}$ assess the different simulation scenarios for $\gamma=0$ and $\gamma=5$ consistently with the other indices (except for $\omega_{1}$ ). The figure $\Phi_{5}$ is an important measure, which refers to a cohort of healthy persons who contract the disease but never get the chance of being treated.

To our knowledge, the disease model in Fig. 1 has only been reported in [7]. In contrast to existing state models with a compartment preceding the diagnosis, typically called preclinical state $[15,16]$, our model includes the possibility of dying from the undiagnosed (preclinical) state. In diabetes, there is a considerably increased mortality from this state [12].

In the literature, there is another index to assess casefinding, the mean sojourn time (MST) in the preclinical phase (see [17] for a review). Usually, a low MST is considered advantageous. However, the MST may be low if
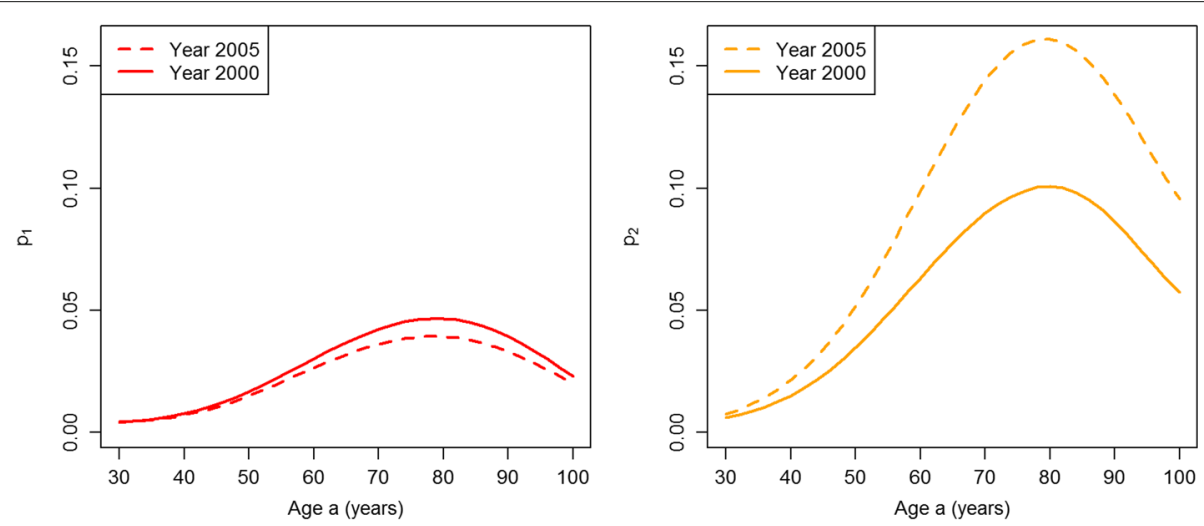

Fig. 7 Prevalences in the improved case-finding scenario. Age-specific prevalences $p_{1}$ (left) and $p_{2}$ (right) in the years $t=2000$ (solid lines) and $t=2005$ (dashed lines) in the scenario $\mathrm{PU}^{-}$ 

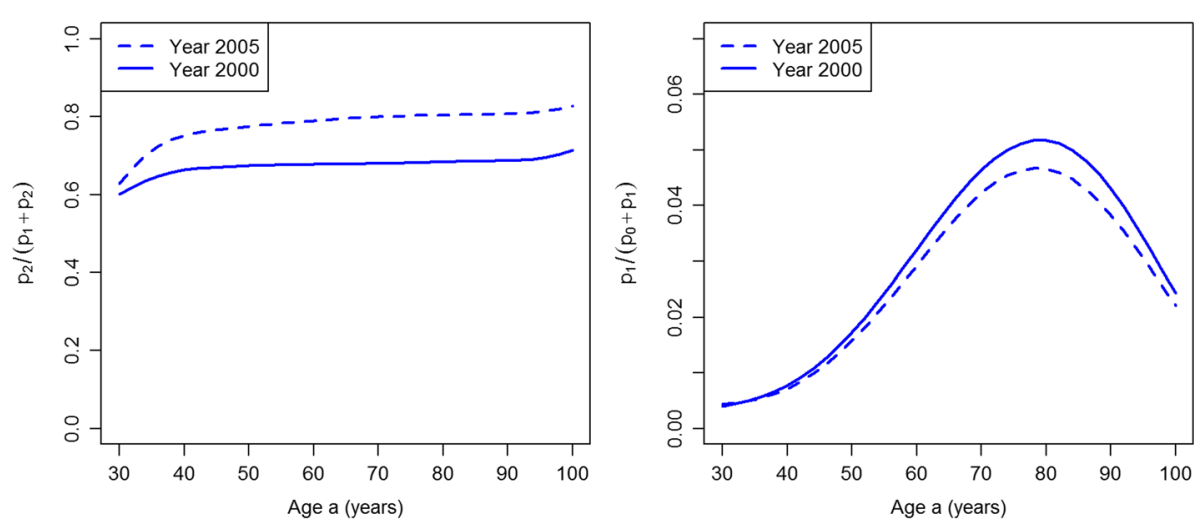

Fig. 8 Prevalence-based indices in the improved case-finding scenario. Age-specific indices of case-finding $\omega_{1}$ (left) and $\omega_{2}$ (right) in the years $t=2000$ (solid lines) and $t=2005$ (dashed lines) in the scenario PU ${ }^{-}$

the mortality from the undiagnosed state is high. Thus, the MST is not an appropriate figure for evaluating casefinding if there is considerable mortality from the undiagnosed (or preclinical) state. Hence, the MST has not been considered in this work.

Apart from the systematic comparison of the different indices, we introduced two new indices $\omega_{2}$ and $\Phi_{\gamma}$. The later quantifies the number of healthy persons who become incident undiagnosed cases and die before ever obtaining a diagnosis. In populations with a high incidence (i.e., large $\lambda_{0}$ ) and a poor case-finding (low $\lambda_{1}$ ), the number $\Phi_{\gamma}$ will be high. This can be observed in the two simulation scenarios. While in the $\mathrm{PU}^{+}$scenario $\Phi_{5}$ increases from 2000 to 2005, there is a large decrease of $\Phi_{5}$ in the $\mathrm{PU}^{-}$scenario. In such cases, it would be interesting to compare this index for different strata of people in the considered population, e.g., low vs. high age, or low versus high socio-economic status.

Compared to the other figures, the measure $\omega_{2}=\frac{p_{1}}{p_{0}+p_{1}}$ has the advantage that it requires prevalence data only.
Prevalences can be obtained from cross-sectional studies. Those measures that include the incidence rates either require costly follow-up data or the application of specialized estimation techniques [7].

The question arises, how the different indices may be surveyed. The prevalence-based metrics $\omega_{1}$ and $\omega_{2}$ can be estimated from cross-sectional surveys that comprise estimation of diagnosed and undiagnosed disease. A variety of cross-sectional studies ask participants about prior diagnoses, for instance, in case of diabetes [18-20] or hypertension [21].

The indices based on the transition rates can be estimated by studies with follow-up information such as cohort studies. A practical demonstration using data from the Health and Retirement Study has been described in a recent manuscript [7], which also illustrates how to deal with statistical uncertainty.

Although the data and examples of this manuscript are given primarily for diabetes, the presented concepts can be applied in other chronic diseases like those mentioned
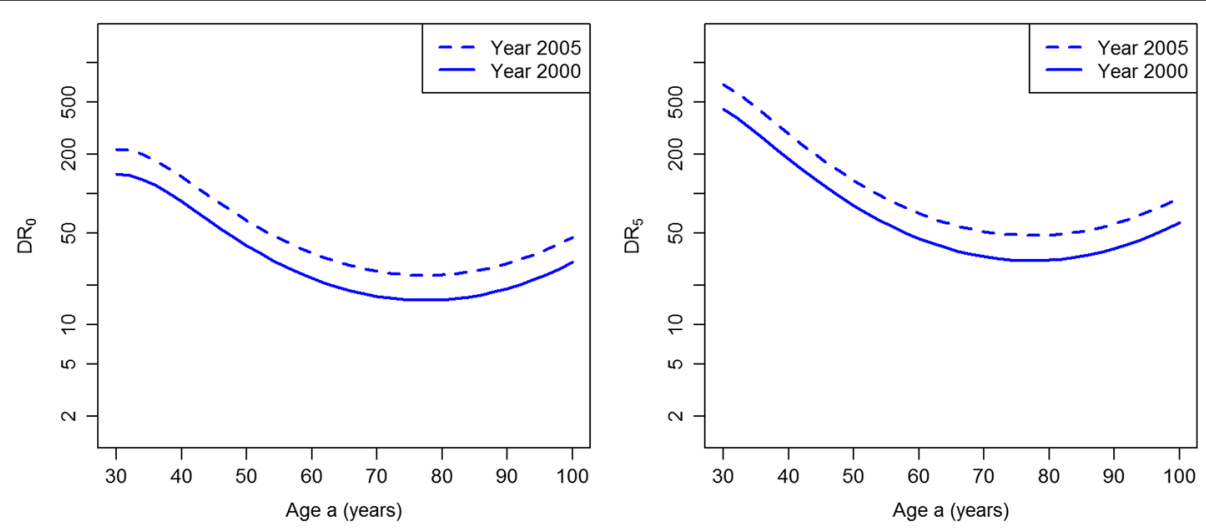

Fig. 9 Detection ratios in the improved case-finding scenario. Age-specific detection ratios DR $\left(\right.$ left) and $\mathrm{DR}_{5}$ (right) in the years $t=2000$ (solid lines) and $t=2005$ (dashed lines) in the scenario $\mathrm{PU}^{-}$ 

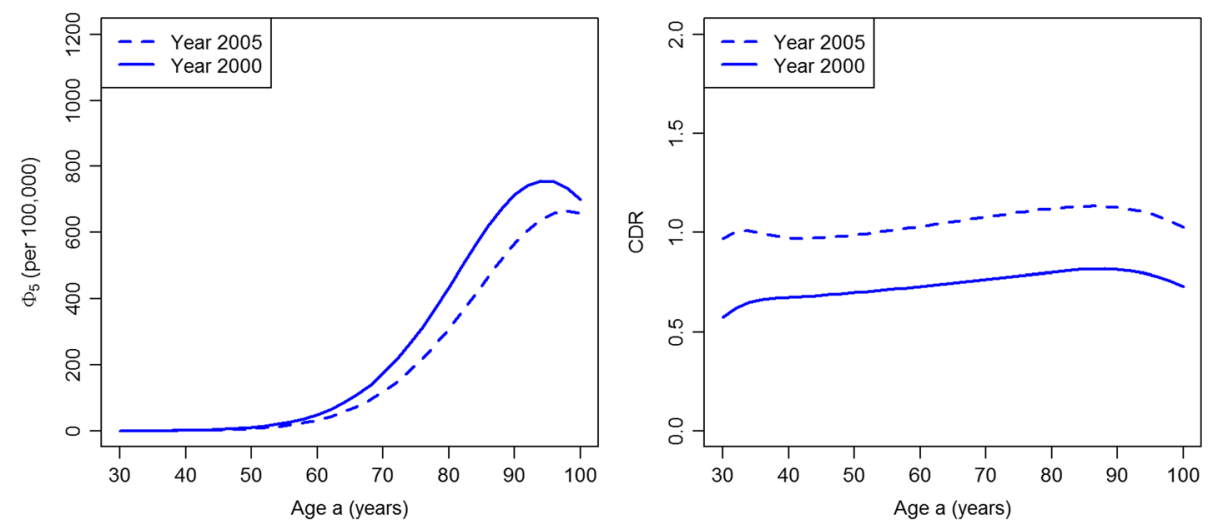

Fig. 10 Number of deaths from the undiagnosed state and case detection rate in the improved case-finding scenario. Age-specific number $\Phi_{5}$ (per 100,000 healthy persons, left) and the case detection rate CDR (right) in the years $t=2000$ (solid lines) and $t=2005$ (dashed lines) in scenario PU ${ }^{-}$

in the introduction. For example, the estimation of $\omega_{1}$ and $\omega_{2}$ in a nationally representative study [21] about hypertension is straight forward.

\section{Conclusion}

This article compiles and compares several indices for case-finding in the field of chronic diseases. To our knowledge, this is the first systematic comparison of indirect surveillance-based metrics for the monitoring of case finding. We found that in assessing casefinding, incidence-based metrics should be preferred, because they gain a deeper insight into the dynamics of the compartment system than prevalence-based indices. Eqs. (1)-(2) show that the prevalences $p_{k}, k=1,2$, depend on all transition rates in the multi-state model, on the incidence rates $\lambda_{\ell}, \ell=0,1$, and also on the mortality rates $\mu_{k}(t, a), k=0,1,2$. In this sense, the prevalences are the result of a complex interplay of the transition rates, both incidence and mortality rates.

Table 2 Summary of the different indices of assessing case-finding

\begin{tabular}{lll}
\hline & \multicolumn{2}{l}{ Prevalence of undiagnosed disease } \\
\cline { 2 - 3 } Index & Increasing $\left(\mathrm{PU}^{+}\right)$ & Decreasing $\left(\mathrm{PU}^{-}\right)$ \\
\hline$\omega_{1}$ & - & + \\
$\omega_{2}$ & - & + \\
$\mathrm{DR}_{0}$ & - & + \\
$\mathrm{DR}_{5}$ & - & + \\
$\mathrm{CDR}$ & $(-)$ & + \\
$\Phi_{5}$ & & $(+)$ \\
\hline
\end{tabular}

A plus (+) or a minus (-) sign denotes whether the index indicates an improvement or a deterioration of case-finding over time. If a plus or minus sign is put in parentheses, the index is indecisive for some ages
Thus, prevalence-based indices of case-finding should be interpreted with caution, because they may lead to inconsistent findings $\left(\omega_{1}\right)$. If prevalence-based metrics have to be used, the new index $\omega_{2}$ is preferable over $\omega_{1}$, because $\omega_{2}$ has shown to be the better index to distinguish the simulated scenarios of increasing or decreasing prevalence of undiagnosed disease.

Indices based on transition rates provide more insights into the system. The detection ratio (DR) provides a necessary and sufficient condition whether the prevalence of undiagnosed disease is increasing or decreasing. Moreover, only the true incidence rate $\lambda_{0}$ indicates if the distributions of the underlying risk factors of the considered chronic disease changes. Thus, if the incidence of a chronic disease is used for surveillance, it is important to always consider the Undiagnosed state. If this state is ignored, and instead the combined state of Normal and Undiagnosed is considered, the rate $\lambda^{\prime}$ surveyed (see Appendix). Changes in the rate $\lambda_{1}$ reflect societal processes like medical progress and changes in patients' or physicians' awareness.

In summary, these simulation analyses identify potential pitfalls of commonly-used indirect measures of case detection. These findings suggest that the $\omega_{2}$ term (proportion of all persons without a diagnosis who have the disease) is a preferable index to the $\omega_{1}$ term (proportion of all cases who have been diagnosed). However, these findings also underscore the need to use more direct estimates of incidence itself and to incorporate more direct estimates of detection into ongoing chronic disease surveillance systems.

\section{Appendix}

The CDR is the proportion of incident cases being diagnosed [8], i.e., the notification rate over the total incidence rate. Let $\lambda^{\prime}$ be the notification rate, which is the number 
of detected cases transiting from the search space to the Diagnosed state per unit time. Thus, the denominator of $\lambda^{\prime}$ is the number of persons in the combined state of Normal and Undiagnosed. In Fig. 1, the rate $\lambda_{1}$ refers to transitions from the Undiagnosed state to the Diagnosed state. Here, the denominator is the number of persons in the Undiagnosed state. Hence, it holds $\lambda^{\prime}=\frac{p_{1}}{p_{0}+p_{1}} \lambda_{1}$. As $\lambda_{0}$ is the overall incidence, it holds

$$
\mathrm{CDR}=\frac{p_{1} \lambda_{1}}{\left(p_{0}+p_{1}\right) \lambda_{0}}=\omega_{2} \mathrm{DR}_{0}
$$

\section{Additional file}

Additional file 1: Scripts for the statistical software R. The zip-file contains the analysis for the simulation. For detailed instructions unzip the file and refer to the readme. txt file. (ZIP $4 \mathrm{~kb}$ )

\section{Abbreviations}

CDR: Case detection rate; $\mathrm{DR}$ : Detection rate ratio; $\mathrm{PU}^{-}$: Improving case-finding; $\mathrm{PU}^{+}$: Worsening case-finding

\section{Acknowledgements}

Not applicable.

\section{Funding}

The authors have not recieved any funding with respect to this work.

\section{Availability of data and materials}

The data used in this article were completely published in [9]. No further collection of individual persons' data has been accomplished. All results can be reproduced from the source files provided as Additional file 1. The source files can be run with the freely available statistical software $R$ (The $R$ Foundation for Statistical Computing).

\section{Authors' contributions}

EWG had the initial idea for this project. RB suggested the indices $\omega_{2}$ and $\Phi_{\gamma}$ RB developed and analysed the simulation and drafted the manuscript. RB, $\mathrm{AH}, \mathrm{DBR}, \mathrm{OK}$ and EWG critically revised the text, gave important intellectual contributions and final approval of the version to be published.

\section{Competing interests}

The authors declare that they have no competing interests.

\section{Consent for publication}

Not applicable as no collection of individual persons' data has been accomplished.

\section{Ethics approval and consent to participate}

Not applicable as no collection of individual persons' data has been accomplished.

\section{Publisher's Note}

Springer Nature remains neutral with regard to jurisdictional claims in published maps and institutional affiliations.

\footnotetext{
Author details

${ }^{1}$ German Diabetes Center, Leibniz Institute for Diabetes Research at the Heinrich-Heine-University Düsseldorf, Institute for Biometry and Epidemiology, Auf'm Hennekamp 65, 40225 Düsseldorf, Germany. ${ }^{2}$ University Hospital at the Heinrich-Heine-University Düesseldorf, Hiller Research Unit for Rheumatology, Moorenstrasse 5, 40225 Düsseldorf, Germany. ${ }^{3}$ Centers for Disease Control and Prevention, Division of Diabetes Translation, Atlanta, Georgia, USA.

${ }^{4}$ German Center for Diabetes Research (DZD), Munich-Neuherberg, Germany.
}

Received: 19 July 2016 Accepted: 23 March 2017

Published online: 11 April 2017

\section{References}

1. Gregg EW, Cadwell BL, Cheng YJ, Cowie CC, Williams DE, Geiss L, Engelgau MM, Vinicor F. Trends in the prevalence and ratio of diagnosed to undiagnosed diabetes according to obesity levels in the US. Diabetes Care. 2004;27(12):2806-812.

2. Coresh J, Byrd-Holt D, Astor B, Briggs J, Eggers P, Lacher D, Hostetter T. Chronic kidney disease awareness, prevalence, and trends among U.S, adults, 1999 to 2000. J Am Soc Nephrol. 2005;16(1):180-8.

3. Ayanian JZ, Zaslavsky AM, Weissman JS, Schneider EC, Ginsburg JA. Undiagnosed hypertension and hypercholesterolemia among uninsured and insured adults in the third National Health and Nutrition Examination Survey. Am J Public Health. 2003;93(12):2051-054.

4. Basu S, Millett C. Social epidemiology of hypertension in middle-income countries determinants of prevalence, diagnosis, treatment, and control in the WHO SAGE study. Hypertension. 2013;62(1):18-26.

5. Lim SS, Vos T, Flaxman AD, Danaei G, Shibuya K, Adair-Rohani H, AlMazroa MA, Amann M, Anderson HR, Andrews KG, et al. A comparative risk assessment of burden of disease and injury attributable to 67 risk factors and risk factor clusters in 21 regions, 1990-2010: a systematic analysis for the Global Burden of Disease Study 2010. Lancet. 2013;380(9859):2224-260.

6. International Diabetes Federation. IDF Diabetes Atlas, 7th ed. Brussles: International Diabetes Federation; 2015.

7. Brinks R, Bardenheier BB, Hoyer A, Lin J, Landwehr S, Gregg EW. Development and demonstration of a state model for the estimation of incidence of partly undetected chronic diseases. BMC Med Res Methodol. 2015;15(1):98

8. Borgdorff M. New measurable indicator for tuberculosis case detection. Emerg Infect Dis. 2004;10(9):1523-28.

9. Carstenson B, Kristensen JK, Ottosen P, Borch-Johnsen K. The Danish National Diabetes Register: Trends in incidence, prevalence and mortality. Diabetologia. 2008:51(12):2187-196.

10. Polyanin AD, Zaitsev VF, Moussiaux A. Handbook of First-Order Partial Differential Equations. Boca Raton: CRC Press; 2001.

11. Dahlquist G, Björck A. Numerical Methods. Englewood Cliffs: Prentice-Hall; 1974.

12. Gordon-Dseagu VLZ, Mindell JS, Steptoe A, Moody A, Wardle J, Demakakos $P$, Shelton NJ. Impaired glucose metabolism among those with and without diagnosed diabetes and mortality: A cohort study using health survey for England data. PLoS ONE. 2015;10(3):0119882. doi:10.1371/journal.pone.0119882.

13. Borg R, Vistisen D, Witte DR, Borch-Johnsen K. Comparing risk profiles of individuals diagnosed with diabetes by OGTT and HbA1c - the Danish Inter99 study. Diabet Med. 2010;27(8):906-10. doi:10.1111/j.1464-5491.2010.03034.x.

14. Egstrup M, Schou M, Gustafsson I, Kistorp CN, Hildebrandt PR, Tuxen CD. Oral glucose tolerance testing in an outpatient heart failure clinic reveals a high proportion of undiagnosed diabetic patients with an adverse prognosis. Eur J Heart Fail. 2011;13(3):319-26. doi:10.1093/eurjhf/hfa216.

15. Lee $\mathrm{S}$, Huang $\mathrm{H}$, Zelen M. Early detection of disease and scheduling of screening examinations. Stat Methods Med Res. 2004;13(6):443-56.

16. Lee SJ, Zelen M. Mortality modeling of early detection programs. Biometrics. 2008;64(2):386-95

17. Uhry Z, Hédelin G, Colonna M, Asselain B, Arveux P, Rogel A, Exbrayat C, Guldenfels C, Courtial I, Soler-Michel P, et al. Multi-state markov models in cancer screening evaluation: a brief review and case study. Stat Methods Med Res. 2010;19(5):463-86.

18. Clair PS, Bugliari D, Campbell N, Chien S, Hayden O, Hurd M, Main R, Miu A, Moldoff M, Panis C, et al. RAND HRS data documentation - Version L. Technical report, Labor \& Population Program, RAND Center for the Study of Aging. 2011.

19. Zhao Y, Hu Y, Smith JP, Strauss J, Yang G. Cohort profile: The China health and retirement longitudinal study (CHARLS). Int J Epidemiol. 2014;43(1):61-68

20. Heidemann C, Du Y, Paprott R, Haftenberger M, Rathmann W, Scheidt-Nave C. Temporal changes in the prevalence of diagnosed diabetes, undiagnosed diabetes and prediabetes: findings from the 
German Health Interview and Examination Surveys in 1997-1999 and 2008-2011. Diabet Med. 2016;33(10):1406-1414. doi:10.1111/dme.13008.

21. Gao Y, Chen G, Tian H, Lin L, Lu J, Weng J, Jia W, Ji L, Xiao J, Zhou Z,

Ran X, Ren Y, Chen T, Yang W. for the China National Diabetes, Group, MDS. Prevalence of hypertension in China: A cross-sectional study. PLOS ONE. 2013;8(6):65938. doi:10.1371/journal.pone.0065938.

Submit your next manuscript to BioMed Central and we will help you at every step:

- We accept pre-submission inquiries

- Our selector tool helps you to find the most relevant journal

- We provide round the clock customer support

- Convenient online submission

- Thorough peer review

- Inclusion in PubMed and all major indexing services

- Maximum visibility for your research

Submit your manuscript at www.biomedcentral.com/submit
Biomed Central 